\title{
Neuroglial plasticity at striatal glutamatergic synapses in Parkinson's disease
}

\author{
Rosa M. Villalba ${ }^{*}$ and Yoland Smith ${ }^{1,2}$ \\ 1 Division of Neuropharmacology and Neurologic Diseases, Yerkes National Primate Research Center, Emory University, Atlanta, GA, USA \\ 2 Department of Neurology, Emory University, Atlanta, GA, USA
}

\section{Edited by:}

James M. Tepper, Rutgers, The State University of New Jersey, USA

\section{Reviewed by:}

Jose L. Lanciego, University of

Navarra, Spain

Paul Bolam, University of Oxford, UK

*Correspondence:

Rosa M. Villalba, Yerkes National Primate Research Center, Emory University, 954 Gatewood Road NE, Atlanta, GA 30329, USA.

e-mail: rvillal@emory.edu
Striatal dopamine denervation is the pathological hallmark of Parkinson's disease (PD). Another major pathological change described in animal models and PD patients is a significant reduction in the density of dendritic spines on medium spiny striatal projection neurons. Simultaneously, the ultrastructural features of the neuronal synaptic elements at the remaining corticostriatal and thalamostriatal glutamatergic axo-spinous synapses undergo complex ultrastructural remodeling consistent with increased synaptic activity (Villalba and Smith, 2011). The concept of tripartite synapses (TS) was introduced a decade ago, according to which astrocytes process and exchange information with neuronal synaptic elements at glutamatergic synapses (Araque et al., 1999a). Although there has been compelling evidence that astrocytes are integral functional elements of tripartite glutamatergic synaptic complexes in the cerebral cortex and hippocampus, their exact functional role, degree of plasticity and preponderance in other CNS regions remain poorly understood. In this review, we discuss our recent findings showing that neuronal elements at cortical and thalamic glutamatergic synapses undergo significant plastic changes in the striatum of MPTP-treated parkinsonian monkeys. We also present new ultrastructural data that demonstrate a significant expansion of the astrocytic coverage of striatal TS synapses in the parkinsonian state, providing further evidence for ultrastructural compensatory changes that affect both neuronal and glial elements at TS. Together with our limited understanding of the mechanisms by which astrocytes respond to changes in neuronal activity and extracellular transmitter homeostasis, the role of both neuronal and glial components of excitatory synapses must be considered, if one hopes to take advantage of glia-neuronal communication knowledge to better understand the pathophysiology of striatal processing in parkinsonism, and develop new PD therapeutics.

Keywords: glia, MPTP, non-human primates, tripartite synapses, astrocyte, striatum, corticostriatal, thalamostriatal

\section{INTRODUCTION}

One of the main neuropathological features of Parkinson's disease $(\mathrm{PD})$ is the degeneration of the nigrostriatal dopaminergic pathway, which induces complex physiological changes within the basal ganglia circuitry, including profound alterations in the activity of the corticostriatal glutamatergic system (Calabresi et al., 1996, 2007; Mallet et al., 2006; Wichmann and Delong, 2007). A major pathological change described in animal models and PD patients is a significant reduction in the density of dendritic spines on striatal medium spiny projection neurons (Ingham et al., 1989; Stephens et al., 2005; Zaja-Milatovic et al., 2005; Day et al., 2006; Deutch et al., 2007; Villalba et al., 2009). Recent evidence from our laboratory demonstrates that the ultrastructural features of pre- and post-synaptic neuronal elements at the remaining corticostriatal and thalamostriatal axo-spinous synapses undergo complex remodeling consistent with increased synaptic activity in the striatum of MPTP-treated parkinsonian monkeys (Villalba and Smith, 2010, 2011). Furthermore, new unpublished findings, recently gathered from these animals demonstrate that perisynaptic astrocytes at cortical and thalamic axo-spinous synapses undergo a significant expansion in parkinsonism. Thus, the goal of this review is to integrate these data, and put forward some hypotheses about the functional significance of ultrastructural reorganization of striatal glutamatergic synapses in parkinsonian condition.

In contrast to the long term belief that astrocytes were merely providing a passive structural support for neurons, evidence obtained during the past decade has challenged this view indicating that astrocytes actively participate in synaptic transmission and neural communication (Araque et al., 1999a,b; Hirrlinger et al., 2004; Schipke and Kettermann, 2004; Haber et al., 2006; Witcher et al., 2007; Araque, 2008; Fellin, 2009; Perea et al., 2009; Araque and Navarrete, 2010; Paixao and Klein, 2010; Perea and Araque, 2010), which led to the concept of tripartite synapses (TS), suggesting that astrocytes integrate, process, and exchange information with pre- and post-synaptic neuronal synaptic elements (Araque et al., 1999b). It is well established that astrocytes also undergo dynamic structural remodeling in response to physiological or pathological changes in synaptic activity in different areas of the CNS, including the hippocampus, cortex, cerebellum, and hypothalamus (Theodosis and MacVicar, 1996; Ventura and Harris, 1999; Jones and Greenough, 2002; Dervan et al., 2004; Slezak et al., 2006; Todd et al., 2006; Theodosis et al., 2008; Buard et al., 2010; Reichenbach et al., 2010). 
In these TS, astrocytes serve as a bridge for non-synaptic communication between neurons, thereby contributing to the processing and integration of synaptic signaling through the formation of complex neuron-glia networks. The use of $3 \mathrm{D}$ reconstruction of synaptic complexes at the electron microscopic level has been instrumental in revealing the intimate structural relationships and plasticity of astroglial processes and neuronal elements at synaptic sites. In the hippocampus and mature neocortex, about half of excitatory axo-spinous glutamatergic synapses are covered by astrocytic processes and considered as TS (Spacek, 1985; Ventura and Harris, 1999; Xu-Friedman et al., 2001). In both the sliced and the intact hippocampus, there is evidence that the surface area of the post-synaptic densities (PSDs) are larger when perisynaptic astroglial processes are present, and presumably those synapses are more effective that those without glial ensheathment (Witcher et al., 2007). Furthermore, 3D reconstructions of hippocampal asymmetric axo-spinous synapses have shown that up-regulation of synaptic activity induces a pronounced increase of the glial coverage of both pre- and post-synaptic structures (Lushnikova et al., 2009). Along the same lines, there is a significant increase in the surface area of glial processes wrapped around glutamatergic synapses in the visual cortex of rats raised in a complex environment (Jones and Greenough, 1996), and in the somatosensory cortex of mice subjected to $24 \mathrm{~h}$ of whisker stimulation (Genoud et al., 2006). Many other studies have confirmed and extended these observations that perisynaptic astroglia undergo dynamic reorganization correlated with increased synaptic plasticity and neuronal activity in different brain regions (Ventura and Harris, 1999; Genoud et al., 2006; Haber et al., 2006; Todd et al., 2006; Witcher et al., 2007, 2010; Lushnikova et al., 2009; Perea et al., 2009; Reichenbach et al., 2010).

\section{FUNCTIONAL SIGNIFICANCE OF TS IN SYNAPTIC COMMUNICATION AND SYNAPTOGENESIS}

As discussed above, the plastic remodeling of glial cells and their active participation in synaptic communication have been demonstrated in different brain regions (Geinisman, 2000; Jourdain et al., 2002; Matsuzaki et al., 2004; Park et al., 2006; Theodosis et al., 2008; Lushnikova et al., 2009; Araque and Navarrete, 2010; Reichenbach et al., 2010). Although the functional significance of these ultrastructural changes remains poorly understood, various mechanisms by which astrocytes could regulate synaptic plasticity have been proposed.

Astrocytes secrete neuroactive substances and actively remove synaptically released neurotransmitters from the synaptic cleft, thereby play an active role in regulating the spillover of transmitter, most particularly glutamate, across synapses or to extrasynaptic receptors (Paixao and Klein, 2010). Two glial EAAT subtypes (GLAST/ EAAT1 and GLT1/EAAT2), distributed on astrocytic membranes in the vicinity of excitatory synapses, indeed, prevent accumulation of extracellular glutamate, overstimulation of glutamate receptors and excitotoxic neuronal death in the CNS (Zhang et al., 2003; Fellin and Carmignoto, 2004). There is evidence for dysregulation of GLT1 expression and increased level of extracellular glutamate in the striatum of MPTP-treated mice (Dervan et al., 2004) and 6-hydroxydopamine-lesioned rats (Chung, et al., 2008; Massie et al., 2010; see below). In addition to their transmitter reuptake properties, astrocytes may also be a source of different gliotransmitters, such as glutamate, ATP, GABA, or D-serine, with different potential neuromodulatory roles of synaptic transmission (see Perea et al., 2009).
Astrocytes are also involved in the formation and maintenance of glutamatergic synapses (Witcher et al., 2007; Barres, 2008; Buard et al., 2010; Pfrieger, 2010). Soluble astrocyte-derived signals such as thrombospondins and cholesterol strongly promote both synapse formation and function (Allen and Barres, 2005; Barres, 2008), indicating that interactions between cell surface molecules and the release of various soluble factors by astroglia may be crucially important to mediate the turnover and enlargement of spines observed with synaptic plasticity. Synaptic astroglia are also critical dynamic regulators of the strength and kinetics of synaptic activity. Astrocytes, indeed, respond to neural stimulation or neurochemical changes in the surrounding extracellular space by extending and modifying their processes (Cornell-Bell et al., 1990), especially around active synapses (Oliet et al., 2001; Hirrlinger et al., 2004).

Astrocytes secrete a number of neurotrophic factors that are potent survival factors for various neuronal populations, including nigrostriatal dopaminergic neurons of the substantia nigra pars compacta $(\mathrm{SNc})$, the main targets of neurodegeneration in PD. The glial cell-line-derived neurotrophic factor (GDNF, neurturin), brain-derived neurotrophic factor (BDNF), and mesencephalic astrocyte-derived neurotrophic factor (MANF) indeed, exert strong influences on the growth, development, and neuroprotection of midbrain dopaminergic neurons and their axonal projections in normal and pathological conditions (Lin et al., 1993; Akerud et al., 2001; Cunningham and Su, 2002; Petrova et al., 2003, 2004; Capowski et al., 2007; Sandhu et al., 2009). Interestingly, valproate and 3-hydroxymorhinan, both of which being protective of midbrain dopaminergic neurons against MPP+-induced neurotoxicity, upregulate the production of neurotrophic factors by astrocytes and reduce reactive microgliosis (Chen et al., 2006).

Together, these findings illustrate a myriad of mechanisms by which astrocytes could actively participate in the development, regulation, protection, and restoration of glutamatergic synapses in the CNS. However, although neuron-glia interactions have been studied in some detail in specific brain regions, very little is known about the substrate and plastic properties of these glio-synaptic networks in the striatum, a basal ganglia structure recognized by its high degree of long term synaptic plasticity and remodeling of glutamatergic synapses in normal and parkinsonian conditions (see below). Thus, as part of an ongoing research program aimed at characterizing the ultrastructural plasticity of cortical and thalamic glutamatergic synapses in the striatum of normal and parkinsonian non-human primates, this review will summarize our recent findings on the synaptic remodeling of axo-spinous synapses in MPTPtreated monkeys (Villalba and Smith, 2010, 2011), and provide new evidence that these morphological changes are accompanied with a significant growth in the extent of glial coverage of striatal glutamatergic synapses in parkinsonian condition.

\section{PLASTICITY OF AXO-SPINOUS STRIATAL GLUTAMATERGIC SYNAPSES IN PARKINSONISM DENDRITIC SPINES AND AXON TERMINALS}

The anatomical substrate and mechanism by which dopamine (DA) regulates striatal glutamatergic activity is complex, activity-dependent and still remains poorly understood (Smith and Bolam, 1990; Bamford et al., 2004; Calabresi et al., 2007; Surmeier et al., 2007; Ballion et al., 2008; Smith and Villalba, 2008; Tian et al., 2010). 
The most common targets where dopaminergic and glutamatergic striatal afferent systems functionally interact are the dendritic spines of striatofugal medium spiny neurons (MSNs; Smith and Bolam, 1990). Most, if not all, cortical innervation of MSNs terminates on dendritic spines (Kemp and Powell, 1971; Raju et al., 2006). Although not as extensive as the corticostriatal system, a significant contingent of thalamic glutamatergic afferents also target dendritic spines and display close relationships with dopaminergic terminals in the striatum (Raju et al., 2006; Moss and Bolam, 2008; Smith et al., 2009a,b). In both animal models of parkinsonism (Figures 1A,B) and PD patients, MSNs lose as much as 30-50\% dendritic spines which, in rats, is accompanied with a similar decrease in the total number of asymmetric glutamatergic synapses (Ingham et al., 1989, 1998; Stephens et al., 2005; Zaja-Milatovic et al., 2005; Day et al., 2006; Deutch et al., 2007; Neely et al., 2007; Scholz et al., 2008; Schuster et al., 2009; Smith et al., 2009b; Villalba et al., 2009; Garcia et al., 2010; Soderstrom et al., 2010). Despite this significant spine loss, some in vivo and in vitro electrophysiological studies suggested an increased activity of the corticostriatal system in parkinsonism (Galarraga et al., 1987; Calabresi et al., 1996; Marti et al., 1999), though this issue remains controversial and appears to be more complex than originally thought (Day et al., 2006; Mallet et al., 2006).

Immunocytochemical studies have demonstrated an increased expression of vesicular glutamate transporter 1 (vGluT1) immunoreactivity, a specific marker of corticostriatal terminals, in the striatum of MPTP-treated parkinsonian monkeys (Raju et al., 2008) and postmortem striatal tissue of PD patients (Kashani et al., 2007). In addition, the striatum of DA-depleted rats contains a larger density of perforated asymmetric synapses (Ingham et al., 1998; Meshul et al., 1999, 2000), a form of structural remodeling associated with increased synaptic efficacy in other brain regions (Greenough et al., 1978; Bertoni-Freddari et al., 1993; Harris and Kater, 1994). Therefore, the overactivity of corticostriatal glutamatergic system described by some authors in rodent models of parkinsonism might result from complex structural and neurochemical changes of glutamatergic axo-spinous synapses in DA-depleted striata.

To further address this issue, we used a 3D electron microscopy reconstruction method to perform a rigorous quantitative analysis of the ultrastructural features of spines specifically targeted by thalamic or cortical afferents in the sensorimotor striatum of normal and MPTP-treated parkinsonian monkeys (Figures 1C1-D2); the results of which having been published in several recent reports from our laboratory (see Smith et al., 2009b; Villalba and Smith, 2010, 2011). In summary, the findings presented in these studies demonstrate that thalamostriatal and corticostriatal afferents target different types of striatal spines, and that both systems undergo complex, and partly different, ultrastructural changes indicative of an increased strength of glutamatergic transmission in parkinsonism (Figures 1E-H). These studies revealed three major ultrastructural features of cortical and thalamic glutamatergic axo-spinous synapses in the primate striatum. First, the dendritic spines targeted by vGluT1-containing corticostriatal terminals are significantly larger and harbor a more extensive PSD than those innervated by vesicular glutamate transporter 2 (vGluT2)-positive thalamostriatal boutons. Second, a subset of vGluT2-positive terminals displays a pattern of multisynaptic connectivity. Third, corticostriatal axo-
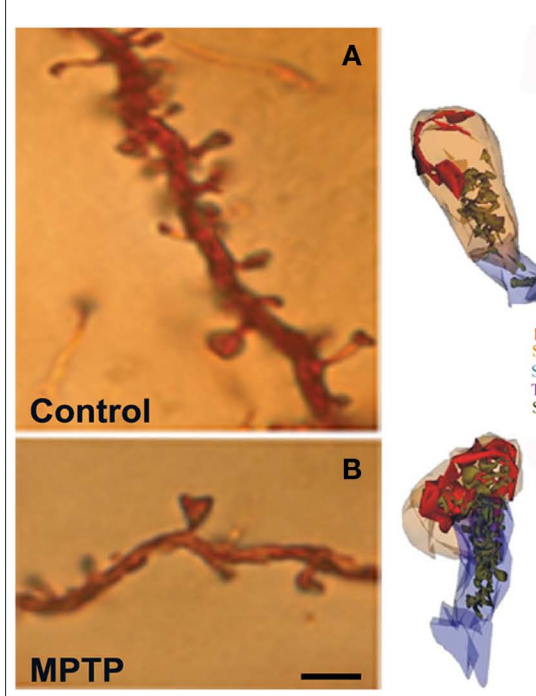

C1
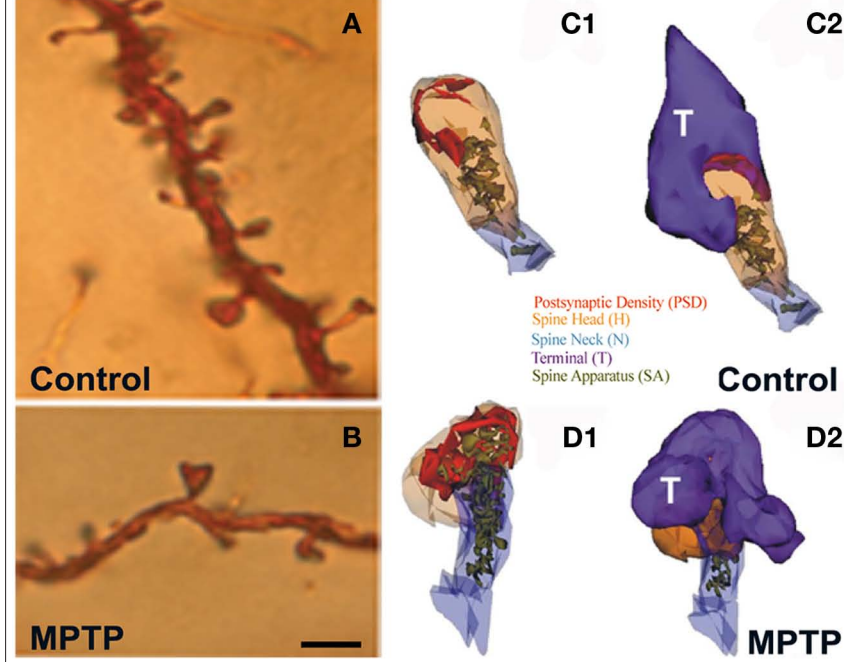

Apparatus (SA)

Control

D1
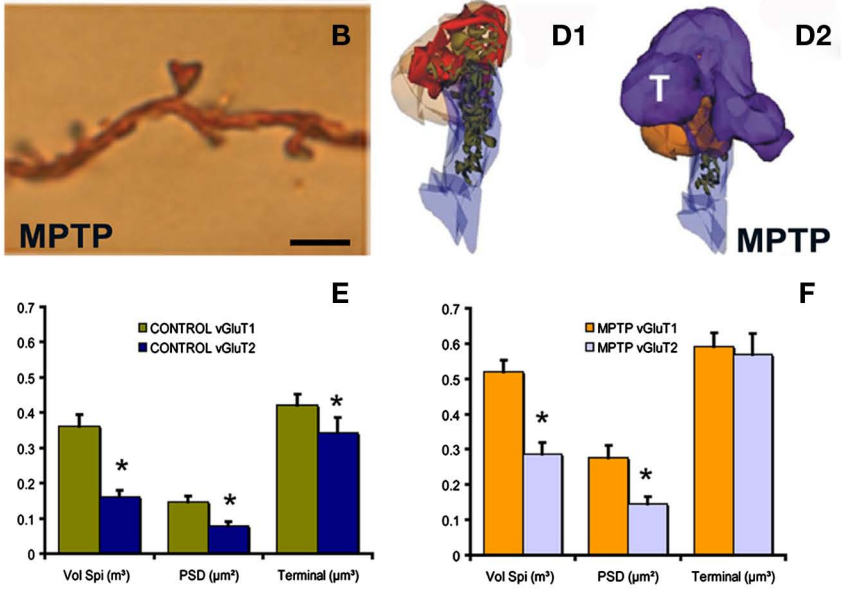

$\mathbf{F}$
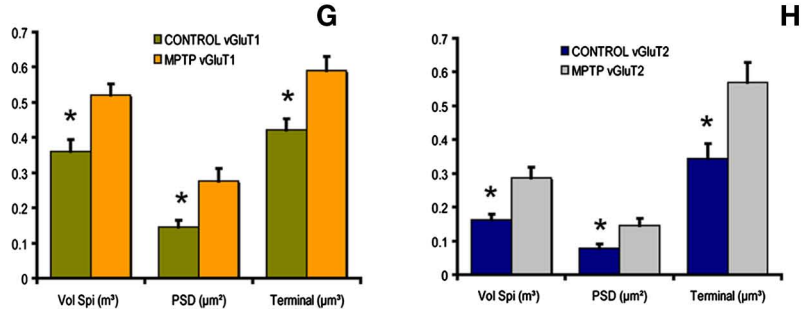

FIGURE 1 | Morphological and ultrastructural changes in striatal MSNs after MPTP treatment. (A,B) Dendrites from Golgi-impregnated MSNs in the caudate nucleus of a control (A) and a MPTP-treated (B) monkey showing the dramatic spine loss (30-50\%) after MPTP treatment. (C1-D2) Threedimension (3D)-reconstructed images of glutamatergic axo-spinous synapses from a control $(\mathbf{C} 1, \mathbf{C} 2)$ and a MPTP-treated $\mathbf{( D 1 , D 2 )}$ monkeys. The spines are partially transparent to better show and compare the complexity and distribution of the spine apparatus (SA) between control and MPTP-treated monkeys. (E,F) Histograms comparing the morphometric measurements (mean \pm SEM; spine volume, PSD area, terminal volume) of structural elements at corticostriatal (vGluT1-positive) and thalamostriatal (vGluT2positive) glutamatergic synapses using the $3 \mathrm{D}$ reconstruction method of serial ultrathin sections collected from 30 axo-spinous synapses in each group from three control and three MPTP-treated animals. The units used for these measurements are indicated in the $X$ axis within parentheses. In control monkeys $(N=3)$, the spine volume (Vol. Sp.), the PSD areas, and the size of pre-synaptic terminals at corticostriatal synapses are significantly larger than those at thalamostriatal synapses $\left[{ }^{*}, t\right.$-test, $P<0.001$ for Vol. Sp. and PSD; $P=0.016$ for terminal (E)]. The same is true for MPTP-treated monkeys $(N=3)$, except for the pre-synaptic terminals that do not show any significant size differences in this condition (F). (G,H) The spine volumes, the PSD areas and the volume of vGluT1- and vGluT2-containing terminals are significantly larger in MPTP-treated parkinsonian monkeys than in controls ( $^{*}, t$-test, $P<0.001$ ). Scale bar in (B) [valid for (A)]: $5 \mu \mathrm{m}$. (see Villalba et al., 2009; Villalba and Smith, 2010, 2011 for more detail.) 
spinous synapses in the sensorimotor putamen of parkinsonian monkeys undergo complex ultrastructural remodeling consistent with increased synaptic activity (larger spine volume, larger PSDs, increased number of PSD perforations, larger pre-synaptic terminal, larger spine apparatus, increased number and decreased volume of terminal mitochondria; Villalba and Smith, 2011). Although some of these plastic changes also characterize vGluT2-positive axo-spinous synapses (larger spine volume, larger PSDs, increased number of PSD perforations), others are specific to cortical afferents (Figures 1E-H). Together with various electrophysiological studies suggesting overactivity of the corticostriatal system in rodent and non-human primate models of parkinsonism (Galarraga et al., 1987; Marti et al., 1999; Gubellini et al., 2002; Liang et al., 2008), these ultrastructural data suggest that striatal MSNs are endowed with a high level of synaptic plasticity that allows, at least early in the disease process, sensorimotor-related information to be properly transmitted and integrated at the striatal level despite a major reduction in the number of corticostriatal synapses (Smith et al., 2009b; Villalba et al., 2009; Villalba and Smith, 2010, 2011).

\section{PERISYNAPTIC ASTROCYTES}

In light of data summarized above (Smith et al., 2009b; Villalba et al., 2009; Villalba and Smith, 2010, 2011) combined with findings from the hippocampus and other brain regions suggesting tight associations between the growth of perisynaptic astrocytic ensheathment and ultrastructural changes in axo-spinous synaptic elements (see above), we have used 3D electron microscopic reconstruction methods to characterize and compare the extent of astrocytic association with cortical (vGluT1-positive) and thalamic (vGluT2-positive) glutamatergic excitatory axo-spinous synapses in the striatum of normal versus MPTP-treated parkinsonian monkeys. The data obtained in this new analysis are summarized below.

\section{Light and electron microscopic analyzes of perisynaptic astrocytes in control and MPTP-treated monkeys}

To address this issue, we first used Golgi impregnation and light microscopy (LM) analysis to examine gross relationships between astrocytic processes and dendritic spines on the surface of MSNs in control and MPTP-treated parkinsonian monkeys (Figure 2). Then, to perform a deeper analysis of the morphological interactions between axo-spinous synapses and perisynaptic astrocytes, we used electron microscopy (EM) 3D reconstruction to quantify and compare the extent of relationships between astrocytic processes and axo-spinous synapses in control and parkinsonian monkeys (Figures 3-6).

LM analysis of astrocytes-dendritic spines relationships. After Golgi impregnation of striatal sections from the post-commissural putamen in three normal and three MPTP-treated parkinsonian monkeys (Vibratome, $60 \mu \mathrm{m}$-thickness; for details on animals and Golgi impregnation see Villalba et al., 2009), we found well impregnated astrocytes intermingled with the spiny dendritic trees of MSNs in both control (Figures 2A,B) and MPTP-treated (Figures 2C,D) animals. The most striking feature of the morphology of protoplasmic astrocytes was the enormous complexity and irregularity of processes arborization, with abundant ramifications often described as bushy or spongiform (Figures 2A,C). These glial

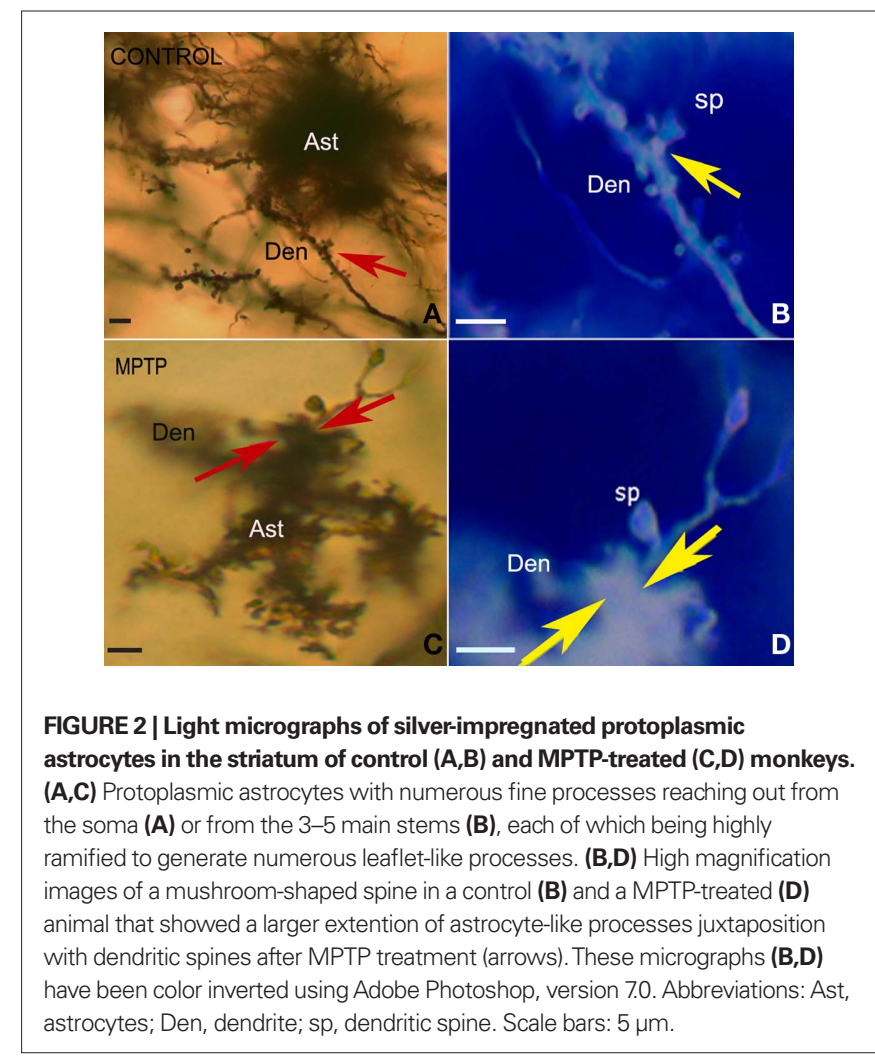

cells harbored rich plexuses of fine processes reaching out from the soma (Figure 2A), or extended 3-5 primary stems that ramified into a large density of fine processes (Figure 2C). At high magnification, some of these processes appeared as thin filopodia- or lamellipodia-like structures, which were insinuated between and around dendritic spines (Figures 2B,D).

Ultrastructural analysis of striatal perisynaptic glia. The following analysis was performed on the same material recently used to study striatal axo-spine plasticity in parkinsonian monkeys (Villalba and Smith, 2011). Methodological details related to animal perfusion, tissue preparation, immunocytochemical procedures, and 3D EM reconstruction methods, described extensively in this previous study, will not be repeated here. We will rather focus our brief methodological description on the procedures used to gather new information on perisynaptic astrocytes. In brief, digital images of vGluT1 - or vGluT2-immunostained tissue sections used to reconstruct individual axo-spinous synapses in our previous study were re-examined, and those complexes associated with perisynaptic astrocytes in single sections were chosen for this analysis. Following screening of the 60 vGluT1- and 60 vGluT2-axo-spinous complexes analyzed in our previous study (Villalba and Smith, 2011), about one-third was found to display significant perisynaptic astrocytic association, thus chosen for the present analysis. In order to avoid any sampling bias, the observer was blinded to the treatment condition.

In single ultrathin sections, perisynaptic astroglial processes were recognized by their irregular shapes, and "clear" cytoplasm as consequence of their lack of most intracellular organelles (pseudocolored 
in blue in Figures 3A,B and 5B). Qualitative comparisons of TS between control and MPTP-treated animals in single ultrathin sections revealed that perisynaptic astrocytes exhibit an interdigitated finger-like morphology in control animals (Figure 3A), while there is an expansion of astrocytic processes to cover a larger extent of the perimeter of axo-spinous complexes after MPTP treatment (Figures 3B, 5A,B).

Three-dimensional reconstructions of striatal tripartite synapses. Through the analysis and digital collection of material gathered from serial ultrathin sections (20-30 sections per grid), individual TS involving vGluT1 - or vGluT2-immunoreactive (IR) terminals in control and MPTP-treated monkeys were reconstructed in 3D using the Reconstruct software application (available at: synapses. clm.utexas.edu). A total of eight vGluT1- and vGluT2-positive TS in normal and MPTP-treated states were chosen for this analysis based on the presence of significant perisynaptic astrocytic processes in close apposition with the axo-spinous complexes. To reconstruct perisynaptic astrocytes, thin astroglial processes were traced through serial sections into larger structures to confirm their

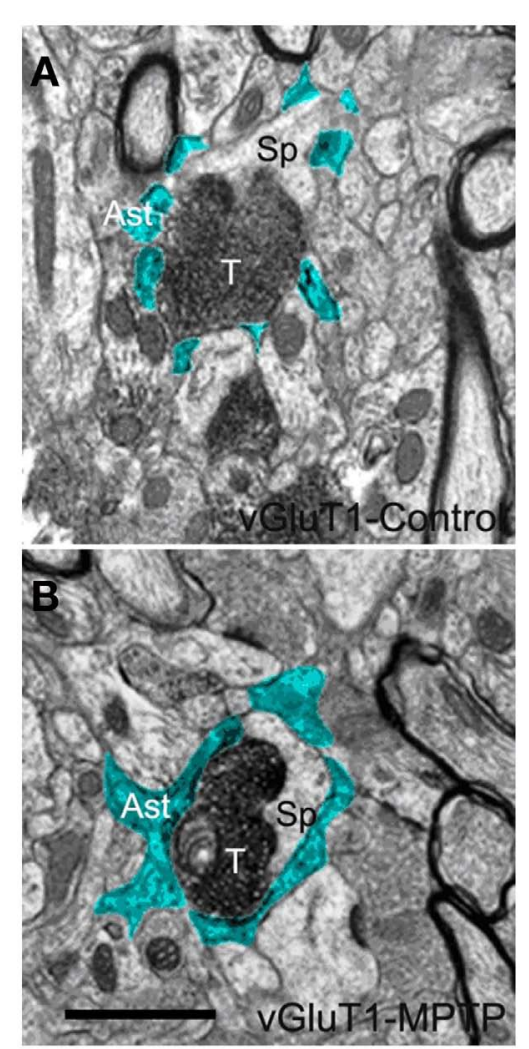

FIGURE 3 | Electron micrographs of a vGluT1-immunoreactive tripartite synapse (TS) in the striatum of a control (A) and a MPTP-treated (B) monkey. Perisynaptic astrocytic processes (Ast) are pseudocolored in blue in $(\mathbf{A}, \mathbf{B})$. These single EM images highlight differences in the extension of the astrocyte wrapping of an axo-spinous synapse between control (A) and MPTP (B) treated animals. Pictures were digitally acquired and imported in TIFF format to Adobe Photoshop (version 7.0; Adobe System, San Jose, CA, USA). Abbreviations: Ast, astrocyte; T, axon terminal; Sp, dendritic spine. Scale bar in (B) [valid for (A)]: $1 \mu \mathrm{m}$. identity and quantify their extent in relation to the pre- and postsynaptic neuronal elements at axo-spinous asymmetric synapses. Briefly, serially digitized electron micrographs of the eight TS that involved vGluT1- or vGluT2-IR terminals in control and MPTPtreated monkeys were converted to TIFF format, imported into Reconstruct, calibrated, and aligned (see Villalba and Smith, 2011 for details). Finally, the individual contours of the astroglial processes were manually traced in each serial electron micrograph using the Reconstruct software. The program calculated the dimensions (surface area and volume) of the perisynaptic astrocytic processes and generated a $3 \mathrm{D}$ representation based on the serial sectioning information.

The analysis of these $3 \mathrm{D}$ reconstructions allowed us to obtain a detailed knowledge of the relationships between axo-spinous complexes and their perisynaptic astrocytic processes. In both TS formed by vGluT1- or vGluT2-immunoreactive axon terminals (Figures 4, 5C-F), highly branched astrocytic processes wrapped themselves around the synapses and formed a nonuniform basket-like structure surrounding the pre- and postsynaptic components of synaptic complexes (Figures 4, 5C-F). Some structural differences were noticed when we compared the extent of perisynaptic glial ensheathment of axo-spinous synapses between control and MPTP-treated monkeys. In control animals, the axo-spinous complexes showed numerous astrocyte-free areas (Figures 4A-C), whereas in MPTP-treated monkeys, the extent of perisynaptic glia was significantly increased, and the appositions between the axo-spinous complex and the astroglial processes were much tighter and continuous forming an extensive barrier around the synaptic complexes (Figures $4 \mathbf{A}^{\prime}-\mathbf{C}^{\prime}, \mathbf{5 C}-\mathbf{F}$ ). These differences between the normal and MPTP condition were seen for both vGluT1- and vGluT2-positive glutamatergic synapses (Figures 4 and 5C-F).

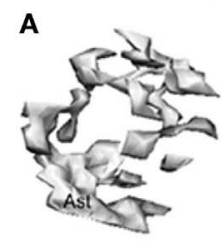

B

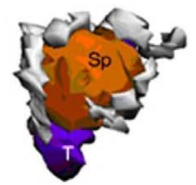

B'

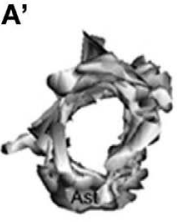

C

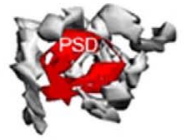

vGluT1- Control

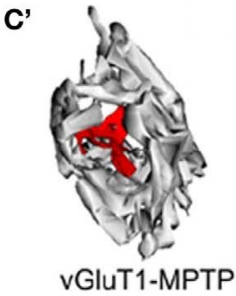

FIGURE 4 |Three-dimensional reconstruction of tripartite synapses (TS) formed by a vGluT1-immunoreactive terminal in the striatum of a control (A-C) and a MPTP-treated $\left(\mathbf{A}^{\prime}-\mathbf{C}^{\prime}\right)$ monkey. (A-C) In the TS of control animals, the perimeters of the axon-spinous interfaces were only partially surrounded by astroglial processes. ( $\left.\mathbf{A}^{\prime}-\mathbf{C}^{\prime}\right)$ TS vGluT1-containing synapses in MPTP-treated animals displayed a large increase in astroglial processes ensheathment. Abbreviations: Ast, astrocyte; PSD, post-synaptic density; Sp, dendritic spine; T, axon terminal. 
To further substantiate these observations, a comparative quantitative assessment of the total surface area of astrocytic processes in direct contact with the 32 reconstructed vGluT1- or vGluT2immunoreactive axo-spinous complexes (16 vGluT1-positive; 16
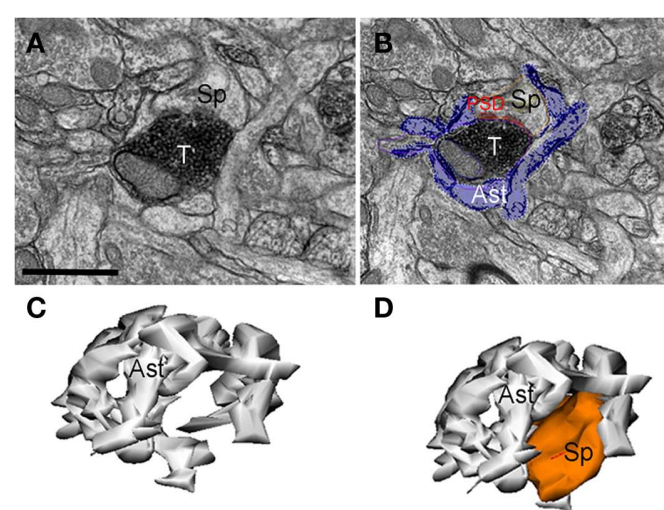

E

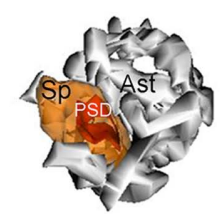

$\mathbf{F}$

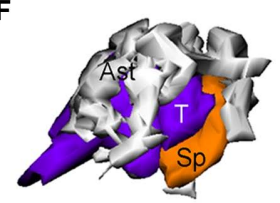

vGluT2-MPTP

FIGURE 5 | Electron micrographs and three-dimensional (3D)reconstruction of a tripartite synapse (TS) formed by a vGluT2immunoreactive terminal in the striatum of a MPTP-treated monkey. $(\mathbf{A}, \mathbf{B})$ Electron micrographs of a vGluT2-immunoreactive TS. Note that in (B) the perisynaptic astrocyte was pseudocolored to help identify the glial processes. (C-F) 3D-reconstruction of the TS demonstrating the tight interactions between the astroglial processes and the axo-spinous vGluT2positive complex. Abbreviations: Ast, astrocyte; PSD, post-synaptic density; $\mathrm{Sp}$, dendritic spine; T, axon terminal. Scale bar in (A) [valid for (B)]: $1 \mu \mathrm{m}$.
vGluT2-positive) was performed between normal and MPTPtreated monkeys (Figure 6; eight TS per group). The statistical analysis ( $t$-test; SigmaPlot; version 11.0) showed that the surface of perisynaptic glia associated with vGluT1- and vGluT2-immunoreactive terminals was significantly larger in MPTP-treated than in control monkeys ( ${ }^{*}$, $t$-test, $P=0.017$ for vGluT1, and $P=0.006$ for vGluT2; Figure 6A). In order to rule out the possibility that this increase in astrocytic surface area was merely due to an increase in the size of the axo-spinous complexes in parkinsonian animals, we calculated the ratio of the volume of the perisynaptic glia over the total volume of the spine and the corresponding axon terminal in each of the 32 TS analyzed (Figure 6B), and found that this ratio was significantly larger for vGluT1- and vGluT2-positive TS in MPTP-treated monkeys than in controls $(P=0.012)$, without any significant difference between the two different populations of terminals ( ${ }^{*}, t$-test, $P=0.049$ for vGluT1 and $P=0.028$ for vGluT2).

\section{CONCLUSION AND FUTURE PERSPECTIVES}

Although there is compelling evidence that astrocytes are integral elements of glutamatergic synaptic complexes, and contribute actively to the regulation and processing of synaptic neural communication, their exact functional role and preponderance of these effects in specific CNS regions remain poorly understood. Findings discussed in this review highlight the significant level of structural plasticity cortical and thalamic glutamatergic axo-spinous synapses are endowed with in the primate striatum, and the ultrastructural compensatory changes that affect remaining glutamatergic synapses in the dopamine-denervated striatum of parkinsonian animals. These results, together with various electrophysiological studies suggesting overactivity of the corticostriatal system in rodent and non-human primate models of parkinsonism (Galarraga et al., 1987; Marti et al., 1999; Gubellini et al., 2002; Liang et al., 2008), illustrate the complex level of synaptic plasticity that governs striatal glutamatergic transmission under normal and parkinsonian conditions.

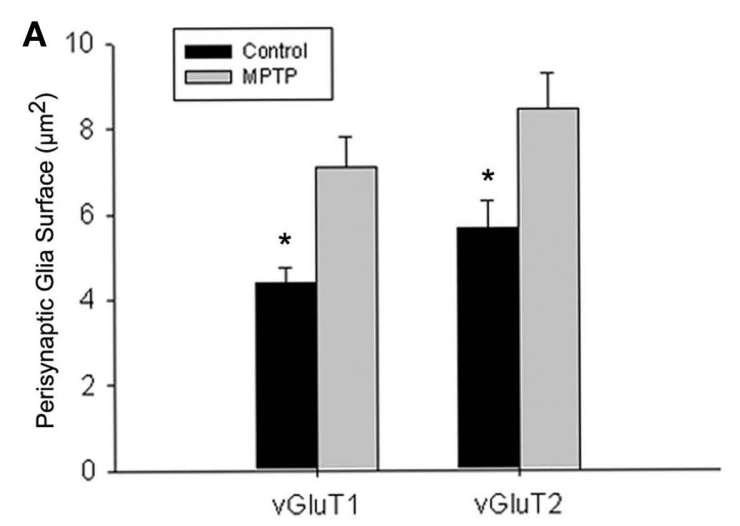

FIGURE 6 | Quantitative analysis of the perisynaptic glia from tripartite synapses (TS) in control and MPTP-treated animals (mean \pm SEM). (A) Histograms comparing the surface area of perisynaptic glia associated with vGluT1- and vGluT2-immunopositive axo-spinous synapses in control $(N=3)$ and MPTP-treated $(N=3)$ monkeys. The surface of the perisynaptic glia was significantly larger (*, $t$-test, $P=0.017$ for vGluT1 and $P=0.006$ for vGluT2) in MPTP-parkinsonian monkeys than in control. (B) Histograms comparing the ratio

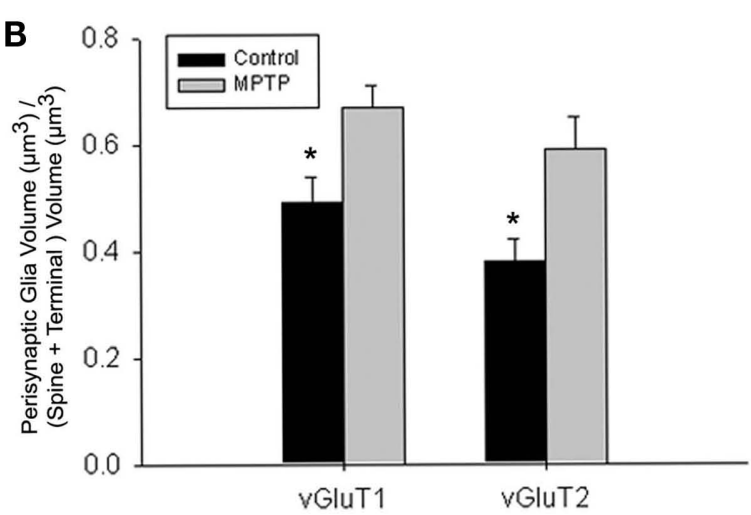

of the volume of the perisynaptic glia over the total volume of the spine and the axon terminals in TS formed by vGluT1- or vGluT2-immunoreactive terminals. This ratio was significantly larger in MPTP than in control condition ( ${ }^{*}, t$-test, $P=0.049$ for vGluT1 and $P=0.028$ for vGluT2). No significant difference was found between TS formed by vGluT1- or vGluT2-immunoreactive terminals. Total number of reconstructed spines $=32,8$ per group. Statistics were performed by using SigmaPlot (version 11.0). 
The increased corticostriatal functions described in MPTPtreated monkeys (Cao et al., 2010) could be the result of a compensatory overactivity of corticostriatal glutamatergic terminals supported by ultrastructural plastic changes described in this and our previous study (Villalba and Smith, 2011). These morphological alterations of axo-spinous cortical and thalamic synapses might underlie the increase in striatal extracellular glutamate concentrations described in rodent models of PD (Lindefors and Ungerstedt, 1990; Meshul et al., 1999; Jonkers et al., 2002; Walker et al., 2009), although these remain controversial (Corsi et al., 2003; Galeffi et al., 2003; Robelet et al., 2004). In addition, the recent in vitro and in vivo data indicating that striatal spine pruning of MSNs in response to dopamine denervation is significantly attenuated by cortical lesions (Neely et al., 2007; Garcia et al., 2010), suggest that these plastic regulatory changes may indirectly contribute to the loss of some striatal spines in PD through increased release and spillover of extracellular glutamate from overactive glutamatergic synapses. Interestingly, the genetic deletion of cerebellin $1(c b \ln 1)$, a gene virtually expressed in all neurons of the thalamic parafascicular nucleus (PF), results in an increased density of striatal spines, suggesting that decreased thalamostriatal drive may be able counteract the effects of DA loss on MSN spine loss, and that the loss of thalamostriatal neurons in PD (see Smith et al., 2009a for a review) may be a compensatory response to striatal DA denervation (Kusnoor et al., 2009, 2010). Although speculative, this possibility is further supported by some reports of downregulation of GLT1 and GLAST in rat and mice models of PD (Holmer et al., 2005; Chung et al., 2008; Massie et al., 2010; but see Lievens et al., 2001).

In addition to structural changes in spine heads, PSDs, and pre-synaptic terminals described in our recent studies (Villalba and Smith, 2010, 2011), and summarized in this review, new data presented in this study indicate that perisynaptic astrocytes also undergo complex morphological changes and significant increase of their association with glutamatergic corticostriatal and thalamostriatal axo-spinous synapses in parkinsonian monkeys. These observations are consistent with the idea of an active participation of glial processes in TS structural plasticity, as previously shown in the hypothalamus (Theodosis et al., 2008) and hippocampus (Ventura and Harris, 1999; Witcher et al., 2007, 2010). However, to further understand the functional role such structural changes may have on the plastic reorganization of glutamatergic axo-spinous complexes, changes in extracellular glutamate concentrations, and spine pruning, future studies assessing potential changes in the content of glutamate transporters and growth factors in peri-

\section{REFERENCES}

Akerud, P., Canals, J. M., Snyder, E. Y., and Arenas, E. (2001). Neuroprotection through delivery of glial cell line-derived neurotrophic factor by neural stem cells in a mouse model of Parkinson's disease. J. Neurosci. 21, 8108-8118.

Allen, N. J., and Barres, B. A. (2005). Signaling between glia and neurons: focus on synaptic plasticity. Curr. Opin. Neurobiol. 15, 542-548.

Araque, A. (2008). Astrocytes process synaptic information. Neuron Glia Biol. 4, 3-10.
Araque, A., and Navarrete, M. (2010). Glial cells in neuronal network function. Philos. Trans. R. Soc. Lond. B Biol. Sci. 365, 2375-2381.

Araque, A., Parpura, V., Sanzgiri, R. P., and Haydon, P. G. (1999a). Tripartite synapses: glia, the unacknowledged partner. Trends Neurosci. 22, 208-215.

Araque, A., Sanzgiri, R. P., Parpura, V., and Haydon, P. G. (1999b). Astrocyteinduced modulation of synaptic transmission. Can. J. Physiol. Pharmacol.77, 699-706.

synaptic astrocytes between normal and MPTP-treated parkinsonian monkeys are warranted. As discussed in the introduction, perisynaptic astrocytes could regulate synaptic transmission by transmitter uptake or release of glutamate and other transmitterrelated neurochemicals. Thus, our findings might underlie a possible change in bi-directional communication between astrocytes and synaptic neuronal elements opening up the possibility for a functional glia-neuronal regulatory loop that could modulate plasticity and efficacy of individual striatal glutamatergic synapses.

In conclusion, the present results add to the growing evidence from other brain regions that both glial and neuronal elements of axo-spinous glutamatergic synapses in the primate striatum are endowed with a high level of structural and, most likely, functional plasticity. However, many unanswered questions remain that must be addressed to better understand the functional role of plastic changes in synaptic glia-neuronal communication in the striatal pathophysiology of parkinsonism. For instance, the prevalence of TS axo-spinous glutamatergic synapses in the striatum in normal and pathological conditions is unknown. Based on data from the hippocampus (Ventura and Harris, 1999; Witcher et al., 2007, 2010), it appears that such a synaptic arrangement is not homogeneous across all excitatory synapses, thereby suggesting that glial influences upon striatal glutamatergic transmission may vary significantly from one synapse to another. This variability in the extent of perisynaptic glia may also make some glutamatergic synapses much more leaky and prone to spill over glutamate in the extracellular medium, thereby activating extrasynaptic receptors, and increase overall striatal activity. Together with our limited understanding of the mechanisms by which astrocytes respond to changes in neuronal activity and extracellular transmitter homeostasis, these issues must be thoroughly investigated if one hopes to take advantage of glia-neuronal communication knowledge to better understand the pathophysiology of striatal processing in parkinsonism, and develop new PD therapeutics that could alter these mechanisms to regulate basal ganglia functions and alleviate disease symptoms.

\section{ACKNOWLEDGMENTS}

The authors thank the Yerkes Center Animal Resources Division for help with the care of MPTP-treated monkeys, and Jean-Francois Pare for his technical assistance with serial electron microscopy sections. This work was supported by the NIH grant R01 NS 037948 and by the NCRR Yerkes Primate Center base grant RR00165.

Ballion, B., Mallet, N., Bezard, E., Lanciego, J. L., and Gonon, F. (2008). Intratelencephalic corticostriatal neurons equally excite striatonigral and striatopallidal neurons and their discharge activity is selectively reduced in experimental parkinsonism. Eur. J. Neurosci. 27, 2313-2321.

Bamford, N. S., Robinson, S., Palmiter, R D., Joyce, J.A., Moore, C., and Meshul, C. K. (2004). Dopamine modulates release from corticostriatal terminals. J. Neurosci. 24, 9541-9552.
Barres, B. A. (2008). The mystery and magic of glia: a perspective on their roles in health and disease. Neuron $60,430-440$.

Bertoni-Freddari, C., Fattoretti, P., Casoli, T., Spagna, C., Meier-Ruge, W., and Ulrich, J. (1993). Compensatory enlargement of synaptic size in aging and senile dementia. Boll. Soc. Ital. Biol. Sper. 69, 57-63.

Buard, I., Steinmetz, C. C., Claudepierre, T., and Pfrieger, F. W. (2010). Glial cells promote dendrite formation and the reception of synaptic input 
in Purkinje cells from postnatal mice. Glia 58, 538-545.

Calabresi, P., Picconi, B., Tozzi, A., and Di Filippo, M. (2007). Dopaminemediated regulation of corticostriatal synaptic plasticity. Trends Neurosci. 30, 211-219.

Calabresi, P., Pisani,A., Mercuri, N. B., and Bernardi, G. (1996). The corticostriatal projection: from synaptic plasticity to dysfunctions of the basal ganglia. Trends Neurosci. 19, 19-24.

Cao, X., Yasuda, T., Uthayathas, S., Watts, R. L., Mouradian, M. M., Mochizuki, H., and Papa, S. M. (2010). Striatal overexpression of DeltaFosB reproduces chronic levodopa-induced involuntary movements. J. Neurosci. 30, 7335-7343.

Capowski, E. E., Schneider, B. L., Ebert, A. D., Seehus, C. R., Szulc, J., Zufferey, R., Aebischer, P., and Svendsen, C. N. (2007). Lentiviral vector-mediated genetic modification of human neural progenitor cells for ex vivo gene therapy.J. Neurosci. Methods 163,338-349.

Chen, P. S., Peng, G. S., Li, G., Yang, S., Wu, X., Wang, C. C., Wilson, B., Lu, R. B., Gean, P. W., Chuang, D. M., and Hong, J. S. (2006). Valproate protects dopaminergic neurons in midbrain neuron/glia cultures by stimulating the release of neurotrophic factors from astrocytes. Mol. Psychiatry 11, 1116-1125.

Chung, E. K., Chen, L. W., Chan, Y.S., and Yung, K. K. (2008). Downregulation of glial glutamate transporters after dopamine denervation in the striatum of 6-hydroxydopamine-lesioned rats. J. Comp. Neurol. 511, 421-437.

Cornell-Bell, A. H., Thomas, P. G., and Smith, S. J. (1990). The excitatory neurotransmitter glutamate causes filopodia formation in cultured hippocampal astrocytes. Glia 3, 322-334.

Corsi, C., Pinna, A., Gianfriddo, M., Melani, A., Morelli, M., and Pedata, F. (2003). Adenosine A2A receptor antagonism increases striatal glutamate outflow in dopaminedenervated rats. Eur. J. Pharmacol. 464, 33-38.

Cunningham, L. A., and Su, C. (2002). Astrocyte delivery of glial cell linederived neurotrophic factor in a mouse model of Parkinson's disease. Exp. Neurol. 174, 230-242.

Day, M., Wang, Z., Ding, J., An, X., Ingham, C.A., Shering, A. F., Wokosin, D., Ilijic, E., Sun, Z., Sampson, A. R., Mugnaini, E., Deutch, A. Y., Sesack, S. R., Arbuthnott, G. W., and Surmeier, D. J. (2006). Selective elimination of glutamatergic synapses on striatopallidal neurons in Parkinson disease models. Nat. Neurosci. 9, 251-259.

Dervan, A. G., Meshul, C. K., Beales, M., McBean, G. J., Moore, C., Totterdell, S., Snyder, A. K., and Meredith, G. E.
(2004). Astroglial plasticity and glutamate function in a chronic mouse model of Parkinson's disease. Exp. Neurol. 190, 145-156.

Deutch, A. Y., Colbran, R. J., and Winder, D. J. (2007). Striatal plasticity and medium spiny neuron dendritic remodeling in parkinsonism. Parkinsonism Relat. Disord. 13(Suppl. 3), S251-S258.

Fellin, T. (2009). Communication between neurons and astrocytes: relevance to the modulation of synaptic and network activity. J. Neurochem. 108, 533-544.

Fellin, T., and Carmignoto, G. (2004). Neurone-to-astrocyte signalling in the brain represents a distinct multifunctional unit. J. Physiol. (Lond.) 559, 3-15.

Galarraga, E., Bargas, J., Martinez-Fong, D., and Aceves, J. (1987). Spontaneous synaptic potentials in dopamine-denervated neostriatal neurons. Neurosci. Lett. 81, 351-355.

Galeffi, F., Bianchi, L., Bolam, J. P., and Della Corte, L. (2003). The effect of 6-hydroxydopamine lesions on the release of amino acids in the direct and indirect pathways of the basal ganglia: a dual microdialysis probe analysis. Eur. J. Neurosci. 18, 856-868.

Garcia, B. G., Neely, M. D., and Deutch, A. Y. (2010). Cortical regulation of striatal medium spiny neuron dendritic remodeling in parkinsonism: modulation of glutamate release reverses dopamine depletion-induced dendritic spine loss. Cereb. Cortex 20, 2423-2432.

Geinisman, Y. (2000). Structural synaptic modifications associated with hippocampal LTP and behavioral learning. Cereb. Cortex 10, 952-962.

Genoud, C., Quairiaux, C., Steiner, P., Hirling, H., Welker, E., and Knott, G. W. (2006). Plasticity of astrocytic coverage and glutamate transporter expression in adult mouse cortex. PLoS Biol. 4, e343. doi: 10.1371/journal.pbio.0040343

Greenough, W. T., West, R. W., and DeVoogd, T. J. (1978). Subsynaptic plate perforations: changes with age and experience in the rat. Science 202, 1096-1098.

Gubellini, P., Picconi, B., Bari, M., Battista, N., Calabresi, P., Centonze, D., Bernardi, G., Finazzi-Agro, A., and Maccarrone, M. (2002). Experimental parkinsonism alters endocannabinoid degradation: implications for striatal glutamatergic transmission. $J$. Neurosci. 22, 6900-6907.

Haber, M., Zhou, L., and Murai, K. K. (2006). Cooperative astrocyte and dendritic spine dynamics at hippocampal excitatory synapses. $J$. Neurosci. 26, 8881-8891.
Harris, K. M., and Kater, S. B. (1994). Dendritic spines: cellular specializations imparting both stability and flexibility to synaptic function. Annu. Rev. Neurosci. 17, 341-371.

Hirrlinger, J., Hulsmann, S., and Kirchhoff, F. (2004). Astroglial processes show spontaneous motility at active synaptic terminals in situ. Eur. J. Neurosci. 20, 2235-2239.

Holmer, H. K., Keyghobadi, M., Moore, C., and Meshul, C. K. (2005). 1-dopainduced reversal in striatal glutamate following partial depletion of nigrostriatal dopamine with 1-methyl4-phenyl-1,2,3,6-tetrahydropyridine. Neuroscience 136, 333-341.

Ingham, C. A., Hood, S. H., and Arbuthnott, G. W. (1989). Spine density on neostriatal neurones changes with 6-hydroxydopamine lesions and with age. Brain Res. 503, 334-338.

Ingham, C.A., Hood, S. H., Taggart, P., and Arbuthnott, G.W. (1998). Plasticity of synapses in the rat neostriatum after unilateral lesion of the nigrostriatal dopaminergic pathway. J. Neurosci. $18,4732-4743$.

Jones, T. A., and Greenough, W. T. (1996). Ultrastructural evidence for increased contact between astrocytes and synapses in rats reared in a complex environment. Neurobiol. Learn. Mem. 65, $48-56$.

Jones, T. A., and Greenough, W. T. (2002). "Behavioral experience-dependent plasticity of glial-neuronal interactions," in The Tripartite Synapses: Glia in Synaptic Transmission, eds A. Volterra, P. J. Magistretti, and P. G. Haydon (Oxford: Oxford University Press), 248-265.

Jonkers, N., Sarre, S., Ebinger, G., and Michotte, Y. (2002). MK801 suppresses the L-DOPA-induced increase of glutamate in striatum of hemi-Parkinson rats. Brain Res. 926, 149-155.

Jourdain, P., Nikonenko, I., Alberi, S., and Muller, D. (2002). Remodeling of hippocampal synaptic networks by a brief anoxia-hypoglycemia. J. Neurosci. 22, 3108-3116.

Kashani,A., Betancur, C., Giros, B., Hirsch, E., and El Mestikawy, S. (2007). Altered expression of vesicular glutamate transporters VGLUT1 and VGLUT2 in Parkinson disease. Neurobiol. Aging $28,568-578$.

Kemp, J. M., and Powell, T. P. (1971). The termination of fibres from the cerebral cortex and thalamus upon dendritic spines in the caudate nucleus: a study with the Golgi method. Philos. Trans. R. Soc. Lond. B Biol. Sci. 262, 429-439.

Kusnoor, S. V., Muly, E. C., Morgan, J. I., and Deutch, A. Y. (2009). Is the loss of thalamostriatal neurons protective in parkinsonism? Parkinsonism Relat. Disord. 155(Suppl. 3), S162-S166.
Kusnoor, S. V., Parris, J., Muly, E. C., Morgan, J.I., and Deutch, A. Y. (2010). Extracerebellar role for Cerebellin 1: modulation of dendritic spine density and synapses in striatal medium spiny neurons. J. Comp. Neurol. 518, 2525-2537.

Liang, L., DeLong, M. R., and Papa, S. M. (2008). Inversion of dopamine responses in striatal medium spiny neurons and involuntary movements. J. Neurosci. 28, 7537-7547.

Lievens, J. C., Salin, P., Nieoullon, A., and Kerkerian-Le Goff, L. (2001). Nigrostriatal denervation does not affect glutamate transporter mRNA expression but subsequent levodopa treatment selectively increases GLT1 mRNA and protein expression in the rat striatum. J. Neurochem. 79 , 893-902.

Lin, L. F., Doherty, D. H., Lile, J.D., Bektesh, S., and Collins, F. (1993). GDNF: a glial cell line-derived neurotrophic factor for midbrain dopaminergic neurons. Science 260, 1130-1132.

Lindefors, N., and Ungerstedt, U. (1990). Bilateral regulation of glutamate tissue and extracellular levels in caudateputamen by midbrain dopamine neurons. Neurosci. Lett. 115, 248-252.

Lushnikova, I., Skibo, G., Muller, D., and Nikonenko, I. (2009). Synaptic potentiation induces increased glial coverage of excitatory synapses in CA1 hippocampus. Hippocampus 19, 753-762

Mallet, N., Ballion, B., Le Moine, C., and Gonon, F. (2006). Cortical inputs and GABA interneurons imbalance projection neurons in the striatum of parkinsonian rats. J. Neurosci. 26, 3875-3884.

Marti, M., Sbrenna, S., Fuxe, K., Bianchi, C., Beani, L., and Morari, M. (1999). In vitro evidence for increased facilitation of striatal acetylcholine release via pre- and postsynaptic NMDA receptors in hemiparkinsonian rats. J. Neurochem. 72, 875-878.

Massie, A., Goursaud, S., Schallier, A., Vermoesen, K., Meshul, C. K., Hermans, E., and Michotte, Y. (2010). Time-dependent changes in GLT-1 functioning in striatum of hemiParkinson rats. Neurochem. Int. 57, 572-578.

Matsuzaki, M., Honkura, N., Ellis-Davies, G. C., and Kasai, H. (2004). Structural basis of long-term potentiation in single dendritic spines. Nature 429, 761-766.

Meshul, C. K., Cogen, J. P., Cheng, H. W., Moore, C., Krentz, L., and McNeill, T. H. (2000). Alterations in rat striatal glutamate synapses following a lesion of the cortico- and/or nigrostriatal pathway. Exp. Neurol. 165, 191-206.

Meshul, C. K., Emre, N., Nakamura, C. M., Allen, C., Donohue, M. K., and 
Buckman,J.F.(1999). Time-dependent changes in striatal glutamate synapses following a 6-hydroxydopamine lesion. Neuroscience 88, 1-16.

Moss, J., and Bolam, J. P. (2008). A dopaminergic axon lattice in the striatum and its relationship with cortical and thalamic terminals. J. Neurosci. 28, 11221-11230.

Neely, M. D., Schmidt, D. E., and Deutch, A. Y. (2007). Cortical regulation of dopamine depletion-induced dendritic spine loss in striatal medium spiny neurons. Neuroscience 149, 457-464.

Oliet, S. H., Piet, R., and Poulain, D. A. (2001).Control of glutamate clearance and synaptic efficacy by glial coverage of neurons. Science 292, 923-926.

Paixao, S., and Klein, R. (2010). Neuronastrocyte communication and synaptic plasticity. Curr. Opin. Neurobiol. 20, 466-473.

Park, J. B., Skalska, S., and Stern, J. E. (2006). Characterization of a novel tonic gamma-aminobutyric acidA receptor-mediated inhibition in magnocellular neurosecretory neurons and its modulation by glia. Endocrinology 147, 3746-3760.

Perea, G., and Araque, A. (2010). Glia modulates synaptic transmission. Brain Res. Rev. 63, 93-102.

Perea, G., Navarrete, M., and Araque, A. (2009). Tripartite synapses: astrocytes process and control synaptic information. Trends Neurosci. 32, 421-431.

Petrova, P., Raibekas, A., Pevsner, J., Vigo, N., Anafi, M., Moore, M. K., Peaire, A. E., Shridhar, V., Smith, D. I., Kelly, J., Durocher, Y., and Commissiong, J. W. (2003). MANF: a new mesencephalic, astrocyte-derived neurotrophic factor with selectivity for dopaminergic neurons. J. Mol. Neurosci. 20, 173-188.

Petrova, P.S., Raibekas, A., Pevsner, J., Vigo, N., Anafi, M., Moore, M. K., Peaire, A., Shridhar, V., Smith, D. I., Kelly, J., Durocher, Y., and Commissiong, J.W. (2004). Discovering novel phenotypeselective neurotrophic factors to treat neurodegenerative diseases. Prog. Brain Res. 146, 168-183.

Pfrieger, F. W. (2010). Role of glial cells in the formation and maintenance of synapses. Brain Res. Rev. 63, 39-46.

Raju, D. V., Ahern, T. H., Shah, D. J., Wright, T. M., Standaert, D. G., Hall, R.A., and Smith, Y. (2008). Differential synaptic plasticity of the corticostriatal and thalamostriatal systems in an MPTP-treated monkey model of parkinsonism. Eur. J. Neurosci. 27, 1647-1658.

Raju, D.V., Shah, D. J., Wright, T. M., Hall, R.A., and Smith, Y. (2006). Differential synaptology of vGluT2-containing thalamostriatal afferents between the patch and matrix compartments in rats. J. Comp. Neurol. 499, 231-243.

Reichenbach, A., Derouiche, A., and Kirchhoff, F. (2010). Morphology and dynamics of perisynaptic glia. Brain Res. Rev. 63, 11-25.

Robelet, S., Melon, C., Guillet, B., Salin, P., and Kerkerian-Le Goff, L. (2004). Chronic L-DOPA treatment increases extracellular glutamate levels and GLT1 expression in the basal ganglia in a rat model of Parkinson's disease. Eur. J. Neurosci. 20, 1255-1266.

Sandhu, J. K., Gardaneh, M., Iwasiow, R., Lanthier, P., Gangaraju, S., RibeccoLutkiewicz, M., Tremblay, R. Kiuchi, K., and Sikorska, M. (2009). Astrocyte-secreted GDNF and glutathione antioxidant system protect neurons against 6OHDA cytotoxicity. Neurobiol. Dis. 33, 405-414.

Schipke, C., and Kettenmann, H. (2004). Astrocytes responses to neuronal activity. Glia 47, 226-232.

Scholz, B., Svensson, M., Alm, H., Skold, K., Falth, M., Kultima, K., Guigoni, C., Doudnikoff, E., Li, Q., Crossman, A. R., Bezard, E., and Andren, P.E. (2008). Striatal proteomic analysis suggests that first L-dopa dose equates to chronic exposure. PLoS ONE 3, e1589. doi: 10.1371/journal.pone.0001589

Schuster, S., Doudnikoff, E., Rylander, D., Berthet, A., Aubert, I., Ittrich, C., Bloch, B., Cenci, M. A., Surmeier, D. J., Hengerer, B., and Bezard, E. (2009). Antagonizing L-type Ca2+ channel reduces development of abnormal involuntary movement in the rat model of L-3,4-dihydroxyphenylalanine-induced dyskinesia. Biol. Psychiatry 65, 518-526.

Slezak, M., Pfrieger, F. W., and Soltys, Z. (2006). Synaptic plasticity, astrocytes and morphological homeostasis. J. Physiol. Paris 99, 84-91.

Smith, A. D., and Bolam, J. P. (1990). The neural network of the basal ganglia as revealed by the study of synaptic connections of identified neurones. Trends Neurosci. 13, 259-265.

Smith, Y., Raju, D., Nanda, B., Pare, J. F., Galvan, A., and Wichmann, T. (2009a). The thalamostriatal systems: anatomical and functional organization in normal and parkinsonian states. Brain Res. Bull. 78, 60-68.

Smith, Y., Villalba, R. M., and Raju, D. V. (2009b). Striatal spine plasticity in Parkinson's disease: pathological or not? Parkinsonism Relat. Disord. 15(Suppl. 3), S156-S161.

Smith, Y., and Villalba, R. (2008). Striatal and extrastriatal dopamine in the basal ganglia: an overview of its ana- tomical organization in normal and Parkinsonian brains. Mov. Disord. 23(Suppl. 3), S534-S547.

Soderstrom, K. E., O'Malley, J. A., Levine, N. D., Sortwell, C. E., Collier, T. J., and Steece-Collier, K. (2010). Impact of dendritic spine preservation in medium spiny neurons on dopamine graft efficacy and the expression of dyskinesias in parkinsonian rats. Eur. J. Neurosci. 31, 478-490.

Spacek, J. (1985). Three-dimensional analysis of dendritic spines. III. Glial sheath. Anat. Embryol. 171, 245-252.

Stephens, B., Mueller, A. J., Shering, A. F., Hood, S. H., Taggart, P., Arbuthnott, G. W., Bell, J. E., Kilford, L., Kingsbury, A. E., Daniel, S. E., and Ingham, C. A (2005). Evidence of a breakdown of corticostriatal connections in Parkinson's disease. Neuroscience 132, 741-754.

Surmeier, D. J., Ding, J., Day, M., Wang, Z., and Shen, W. (2007). D1 and D2 dopamine-receptor modulation of striatal glutamatergic signaling in striatal medium spiny neurons. Trends Neurosci. 30, 228-235.

Theodosis, D. T., and MacVicar, B. (1996) Neurone-glia interactions in the hypothalamus and pituitary. Trends Neurosci. 19, 363-367.

Theodosis, D. T., Poulain, D. A., and Oliet, S. H. (2008). Activity-dependent structural and functional plasticity of astrocyte-neuron interactions. Physiol. Rev. 88, 983-1008.

Tian, X., Kai, L., Hockberger, P. E., Wokosin, D. L., and Surmeier, D. J. (2010). MEF-2 regulates activitydependent spine loss in striatopallidal medium spiny neurons. Mol. Cell. Neurosci. 44, 94-108.

Todd, K. J., Serrano, A., Lacaille, J. C., and Robitaille, R. (2006). Glial cells in synaptic plasticity. J. Physiol. Paris 99, 75-83.

Ventura, R., and Harris, K. M. (1999). Three-dimensional relationships between hippocampal synapses and astrocytes. J. Neurosci. 19, 6897-6906.

Villalba, R. M., Lee, H., and Smith, Y. (2009). Dopaminergic denervation and spine loss in the striatum of MPTP-treated monkeys. Exp. Neurol. 215, 220-227.

Villalba, R. M., and Smith, Y. (2010). Striatal spine plasticity in Parkison's disease. Front. Neuroanat. 4:133. doi 10.3389/fnana.2010.00133

Villalba, R. M., and Smith, Y. (2011). Differential structural plasticity of corticostriatal and thalamostriatal axospinous synapses in MPTP-treated parkinsonian monkeys. J. Comp. Neurol. 519, 989-1005.

Walker, R. H., Koch, R. J., Sweeney, J. E., Moore, C., and Meshul, C.
K. (2009). Effects of subthalamic nucleus lesions and stimulation upon glutamate levels in the dopaminedepleted rat striatum. Neuroreport 20, 770-775.

Wichmann, T., and Delong, M. R. (2007). Anatomy and physiology of the basal ganglia: relevance to Parkinson's disease and related disorders. Handb. Clin. Neurol. 83, 1-18.

Witcher, M. R., Kirov, S. A., and Harris, K. M. (2007). Plasticity of perisynaptic astroglia during synaptogenesis in the mature rat hippocampus. Glia $55,13-23$.

Witcher, M. R., Park, Y. D., Lee, M. R., Sharma, S., Harris, K. M., and Kirov, S. A. (2010). Three-dimensional relationships between perisynaptic astroglia and human hippocampal synapses. Glia 58, 572-587.

Xu-Friedman, M. A., Harris, K. M., and Regehr, W. G. (2001). Threedimensional comparison of ultrastructural characteristics at depressing and facilitating synapses onto cerebellar Purkinje cells. J. Neurosci. 21, 6666-6672.

Zaja-Milatovic, S., Milatovic, D., Schantz, A. M., Zhang, J., Montine, K. S., Samii, A., Deutch, A. Y., and Montine, T. J. (2005). Dendritic degeneration in neostriatal medium spiny neurons in Parkinson disease. Neurology 64, 545-547.

Zhang,J.M., Wang, H. K., Ye, C. Q., Ge, W., Chen, Y., Jiang, Z. L., Wu, C. P., Poo, M. M., and Duan, S. (2003). ATP released by astrocytes mediates glutamatergic activity-dependent heterosynaptic suppression. Neuron 40, 971-982.

Conflict of Interest Statement: The authors declare that the research was conducted in the absence of any commercial or financial relationships that could be construed as a potential conflict of interest.

Received: 22 April 2011; paper pending published: 12 May 2011; accepted: 02 August 2011; published online: 23 August 2011

Citation: Villalba RM and Smith Y (2011) Neuroglial plasticity at striatal glutamatergic synapses in Parkinson's disease. Front. Syst. Neurosci. 5:68. doi: 10.3389/ fnsys. 2011.00068

Copyright $(\odot) 2011$ Villalba and Smith. This is an open-access article subject to a nonexclusive license between the authors and Frontiers Media SA, which permits use, distribution and reproduction in other forums, provided the original authors and source are credited and other Frontiers conditions are complied with. 